MODELING, IDENTIFICATION AND CONTROL, 2001, VOL. 22, NO. 2, 103-118

doi:10.4173/mic.2001.24

\title{
An integration scheme for stiff solid-gas reactor models*
}

\author{
BJARNE A. FOSS $\uparrow$ and STEIN O. WASBØ $\ddagger$ \\ Keywords: Stiff systems, numerical integration, industrial application
}

\begin{abstract}
Many dynamic models encounter numerical integration problems because of a large span in the dynamic modes. In this paper we develop a numerical integration scheme for systems that include a gas phase, and solid and liquid phases, such as a gas-solid reactor. The method is based on neglecting fast dynamic modes and exploiting the structure of the algebraic equations. The integration method is suitable for a large class of industrially relevant systems. The methodology has proven remarkably efficient. It has in practice performed excellent and been a key factor for the success of the industrial simulator for electrochemical furnaces for ferro-alloy production.
\end{abstract}

\section{Motivation and literature survey}

There are many dynamic models that encounter numerical integration problems due to the fact that the span in dynamic modes is very large. Some examples are atmospheric phenomena models such as air pollution models; process models for distillation columns, catalytic processes and solid-gas reactors; multi-body models that include both rigid and elastic modes as encountered in detailed models of satellites; and dynamic models of power distribution systems e.g. an electric grid, or fluid or gas network. See de Swart et al. (1999) for a collection of some of these types of models. In the case of a catalytic process the span in dynamic modes stems from the fact that a commercial catalyst may have a life-time of 1-5 years while the reaction(s) that the catalyst catalyzes may be in the order of seconds or minutes. Likewise, in a solid-gas reactor the residence time of the gas phase may be in the order of seconds while the residence time of the solid phase(s) may be in the order of hours. Dynamic models that stem from spatial discretizing of a distributed model representation, e.g. partial differential equations, often exhibit a large span in dynamic modes. A typical example of the latter is the process model of a Kamyr digester, i.e. a commercial pulping process, described in Michelsen and Foss (1996).

Dynamic models that exhibit a large span in dynamic modes are usually denoted stiff models. For linear systems this property can be checked by computing the ratio of the largest absolute eigenvalue to the smallest absolute eigenvalue of the system matrix. There is no equivalent test for nonlinear systems. One descriptive definition is, however, given in Lambert (1991):

If a numerical method with a finite region of absolute stability, applied to a system with any initial conditions, is forced to use in a certain interval of integration a step-length which is excessively small in relation to the smoothness of the exact solution in that interval, then the system is said to be stiff in that interval.

*Reprinted from Computer Methods in Applied Mechanics and Engineering 2001, with permission from Elsevier Science.

†Department of Engineering Cybernetics, Norwegian University of Science and Technology, Trondheim, Norway. E-mail: Bjarne.Foss@itk.ntnu.no

$\ddagger$ Eramet Norway A.S. E-mail: Stein.Wasbo@eramet-mn-no.com 
This definition pinpoints the major problem with stiff models, the fact that the computational effort to compute a solution may be prohibitive.

In this study we will assume a model structure as a set of differential-algebraic equations (DAEs) with known initial conditions.

$$
\begin{aligned}
\dot{x} & =f(x, y, z) \\
\dot{y} & =g(x, y, z) \\
h(x, y, z) & =0 \\
x\left(t_{0}\right) & =x_{0} \\
y\left(t_{0}\right) & =y_{0}
\end{aligned}
$$

$x$ and $y$ are the system states, and $z$ is an auxiliary variable. $\dot{x}$ denotes the derivative with respect to time. In a chemical process model $z$ might include the partial equilibrium pressure for the modelled reactions, the partial pressures and enthalpies, or $z$ might include the temperature if the inner energy is used as a state variable. The functions $f, g$ and $h$ are assumed to be Lipschitz continuous to assure existence and uniqueness of a solution of equations (1)-(3) on a finite time interval $t_{0}$ to $t_{1}$, i.e. $\left(x(t), y(t), z(t), t \in\left[t_{0}, t_{1}\right]\right)$. The states are divided into two parts, $x$ and $y$, so as to be able to treat these states independently. In the sequel we assume that we can classify the dynamic modes into slow and fast dynamic modes associated with $x$ and $y$, respectively.

There exists many integration methods for handling stiff systems. The main approaches are:

- Neglect the fast dynamic modes by converting the differential equations for the fast dynamics into algebraic equations.

- Slower the dynamics of fast modes.

- Use an integration method for stiff systems.

The first method changes equations (1)-(3) since equation (2) becomes an algebraic equation.

$$
g(x, y, z)=0
$$

This implies that the DAEs become non-stiff. It is usually impossible to compute $y$ and $z$ prior to the numerical integration of equation (1). Hence, the computational load may far exceed the computational load to integrate equation (1), since it is necessary to iterate between equation (1), and the two algebraic equations (6) and (3). A disadvantage of this approach is that model errors are deliberately introduced into the model.

It is a well established technique to slow down the dynamics of the fast modes. As an example the fast dynamic modes are typically linked to the gas phase in the case of chemical reactors. By increasing the residence time for the gas phase the integration load will be lowered since the DAEs become less stiff. A disadvantage of this approach is, again, that model errors are deliberately introduced into the model. In particular the effects of the interaction between the "slowed-down" gas phase dynamics and the rest of the model can be detrimental.

The disadvantage of having to use an integration method for stiff systems is the fact that these methods require a high computational load. Typically an implicit 
solver is used, e.g. an implicit Runge-Kutta method, or a backwards-difference algorithm. Numerical solvers for stiff systems are treated in detail in Aitken (ed.) (1985), Lambert (1991) and Hairer and Wanner (1991).

\section{Problem formulation}

In this paper we will study systems that include a gas phase, and solid and liquid phases. The systems are spatially discretized into compartments with homogeneous conditions. This is shown in Figure 1 in which the system is discretized into $N$ compartments. We make the following assumptions:

A1 There is a unidirectional gasflow in the vessel.

A2 There is a unidirectional flow of all liquid and solid phases.

A3 External flows can be injected or withdrawn from arbitrary places on the surface of the vessel.

A4 The vessel can be modelled with sufficient accuracy by dividing it into compartments with homogeneous conditions.

We will develop an integration scheme, applicable to the model structure defined above, in which, to the authors' knowledge, the computational load for numerical integration is dramatically decreased compared to all conventional methods. This will be achieved by explicitly solving the algebraic equations, $\dot{y}=g(x, y, z)=0$ and equation (3) in an efficient way, prior to the integration of equation (1).

The methodology is based on the following procedure:

1. The gas dynamics, i.e. the holdup or concentration of gas species, are neglected by converting the differential equations for the fast dynamics into algebraic equations. This is done by associating $y$ with the holdup or concentration of gas species.

2. $y$ and $z$ will be computed prior to the integration of equation (1) by exploiting the structure of the model equations.

3. The dominant dynamics equation (1) are integrated using a numerical integration method for non-stiff systems.

The critical issue is to establish an efficient method for computing $y$ and $z$ since stage 3 involves the use of some standard integration method for non-stiff systems. Such integration methods are not computationally intensive.

\section{Method}

In this section we develop a numerically efficient method for solving equations (6) and (3), i.e. compute $y$ and $z$. Some additional assumptions will be made in order to establish the algorithm. The assumptions are discussed, and finally the algorithm is analyzed.

\subsection{Theory}

We start the derivation by viewing compartment $i$ in Figure 1. It exchanges gas components through an inflow $F_{g, i+1}$ from compartment $i+1$ and an outflow $F_{g, i}$ to compartment $i-1$. It exchanges solid and liquid components through an inflow $F_{\mathrm{s}, i-1}$ from compartment $i-1$ and an outflow $F_{\mathrm{s}, i}$ to compartment $i+1$. Further, 


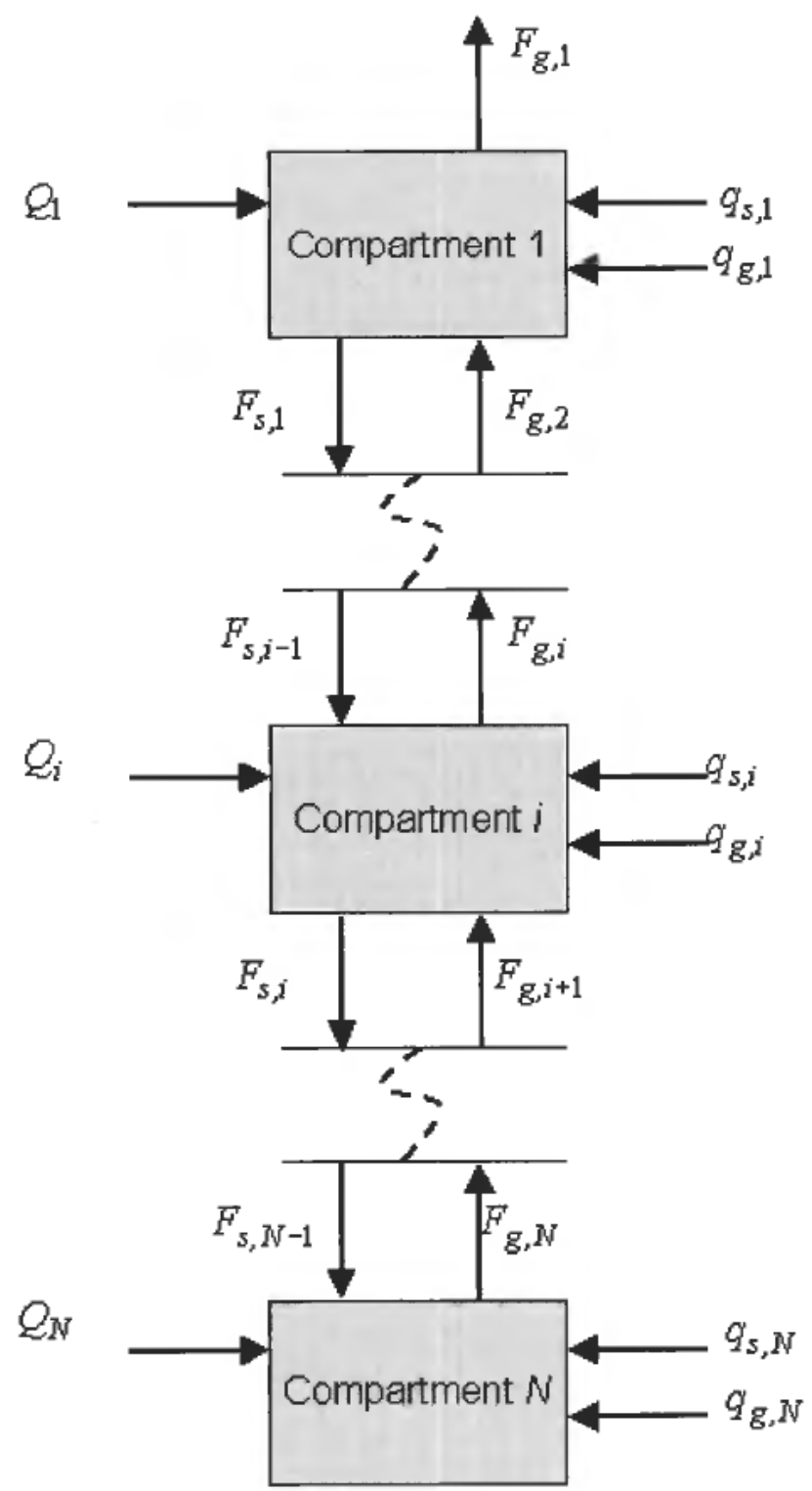

Figure 1. A system is spatially discretized into $N$ compartments. Each compartment can interact with the environment through external mass and energy flow.

the compartment may exchange mass with the surroundings $q_{s, i}$ and $q_{g, i}$. There may also be a pure energy transport $Q_{i}$ between the compartment and the external environment. $V_{i}$ is the volume of compartment $i$.

The component mass balances (in moles) for compartment $i$ are given by

$$
\begin{aligned}
& \dot{n}_{s, i}=F_{s, i-1}-F_{s, i}+q_{s, i}+S_{s} r_{i} \\
& \dot{n}_{g, i}=-F_{g, i}+F_{g, i+1}+q_{g, i}+S_{g} r_{i} \\
& n_{s, i} \in R^{\alpha}, n_{g, i} R^{\beta}, r_{i} R^{\gamma}
\end{aligned}
$$


where $S_{s}$ and $S_{g}$ denote the stoichiometry matrices for the reactions and phase transistions, e.g. melting of a solid or vaporisation of a liquid, that take place within compartment $i$.

The energy balance for the compartment, provided shaft work is neglected, is given by

$$
\begin{aligned}
\dot{U}_{i}= & H_{s}^{T}\left(T_{i-1}\right) F_{s, i-1}-H_{s}^{T}\left(T_{i}\right) F_{s, i}+H_{g}^{T}\left(T_{i+1}\right) F_{g, i+1}-H_{g}^{T}\left(T_{i}\right) F_{g, i} \\
& +H_{s}^{T}\left(T_{i}, T_{e}\right) q_{s, i}+H_{g}^{T}\left(T_{i}, T_{e}\right) q_{g, i}+\Delta H^{T}\left(T_{i}\right) r_{i}+Q_{i}
\end{aligned}
$$

$H_{s}, H_{g}$ denote the enthalpy content for the solids and liquid phases, and gas phase. $\Delta H$ denotes the reaction enthalpy. If there is an inflow of gas, $H_{g}$ should be computed on the basis of an external temperature. If there is an outflow of gas, $H_{g}$ should be computed on the basis of the internal temperature $T_{i}$. Likewise, $H_{s}$ can be computed on the basis of an external temperature or the internal temperature.

We focus on the gas component balance equation (8) and make the following assumptions:

A5 The gas dynamics are neglected, i.e. $\dot{n}_{g, i}=0$.

A6 The reaction kinetics are independent of or linear in the partial pressure of one of the gas components.

A7 The gas phase can be treated like an ideal gas.

The gas balance equation is rewritten component-wise using assumption A5.

$$
F_{g j, i}=F_{g j, i+1}+q_{g j, i}+\sum_{k=1}^{\gamma} s_{g j, k} r_{k, i}, j \in\{1, \ldots, \beta\}
$$

Assumption A6 implies that the reaction rates are given by

$$
r_{k, i}=f_{k}\left(n_{s, i}, T_{i}\right)\left(p_{l(k), i}-\bar{p}_{l(k)}\left(T_{i}\right)\right), k \in\{1, \ldots, \gamma\}
$$

This implies that reaction $k$ is linear in the partial pressure of gas component $l(k) . \bar{p}_{l(k)}\left(T_{i}\right)$ is the equilibrium partial pressure for reaction $k$ at temperature $T_{i}$. A typical structure for $f_{k}$ is $f_{k}\left(n_{s, i}, T_{i}\right)=a_{k} n_{s, j, i} e^{-E_{k} / R T_{i}}$ where $a_{k}$ usually is denoted the frequency factor and $e^{-E_{k} / R T_{i}}$ is the Arrhenius term. $E_{k}$ and $R$ are denoted, the activation energy and the ideal gas constant, respectively. For basic theory on reaction kinetics we refer to Fogler (1986) and Levenspiel (1972).

From assumption A7 we make use of Dalton's law on partial pressure within a confined volume,

$$
\frac{p_{j, i}}{p_{\Sigma, i}}=\frac{n_{g j, i}}{\Sigma_{j=1}^{\beta} n_{g j, i}}, j \in\{1, \ldots, \beta\}
$$

and on Amagat's law for partial volumes within a confined volume.

$$
\frac{V_{g j, i}}{V_{g, i}}=\frac{n_{g j, i}}{\Sigma_{j=1}^{\beta} n_{g j, i}}, j \in\{1, \ldots, \beta\}
$$

Combining equations (12) and (13), and noting that the ratio of the gas flow from compartment $i$ equals the ratio of the partial volumes, gives the following relation:

$$
\frac{F_{g j, i}}{(1, \ldots, 1) \cdot F_{g j, i}}=\frac{p_{j, i}}{p_{\Sigma, i}}, j \in\{1, \ldots, \beta\}
$$

denotes the scalar product. 
The ratio of the component-wise outflow to the total outflow equals the ratio of the partial pressure to the pressure. Equation (14) can be rewritten by using (10).

$$
\begin{aligned}
\left(F_{g j, i+1}+q_{g j, i}+\sum_{k=1}^{\gamma} s_{g j, k} r_{k, i}\right) p_{\Sigma, i}= & p_{j, i}(1, \ldots, 1) \\
& \cdot\left(F_{g j, i+1}+q_{g j, i}+\sum_{k=1}^{\gamma} s_{g j, k} r_{k, i}\right)
\end{aligned}
$$

Inserting equation (11) gives:

$$
\begin{aligned}
& \left(F_{g j, i+1}+q_{g j, i}+\sum_{k=1}^{\gamma} s_{g j, k} f_{k}\left(n_{s, i}, T_{i}\right)\left(p_{l(k), i}-\bar{p}_{l(k)}\left(T_{i}\right)\right) p_{\Sigma, i}\right. \\
= & p_{j, i}(1, \ldots, 1) \cdot\left(F_{g, i+1}+q_{g, i}\right)+p_{j, i} \sum_{j=1}^{\beta} \sum_{k=1}^{\gamma} s_{g j, k} f_{k}\left(n_{s, i}, T_{i}\right)\left(p_{l(k), i}-\bar{p}_{l(k)}\left(T_{i}\right)\right)
\end{aligned}
$$

We introduce the following assumptions:

A8 The pressure $p_{\Sigma, i}$ is known.

A9 The gas inflow $F_{g j, i+1}$ is known.

A10 The external gasflow $q_{g, i}$ is known.

A11 The solids and liquid component mass $n_{s, i}$ are known.

A12 The temperature $T_{i}$ is known.

The five items above imply that only $p_{i}$ is unknown in equation (16). Inspection of equation (16) shows the following:

- The terms $F_{g j, i+1}$ and $q_{g j, i}$ are independent of $p_{i}$.

- The term $\Sigma_{k=1}^{\gamma} s_{g j, k} r_{k, i}=\Sigma_{k=1}^{\gamma} s_{g j, k} f_{k}\left(n_{s, i}, T_{i}\right)\left(p_{l(k), i}-\bar{p}_{l(k)}\left(T_{i}\right)\right)$ is linear in $p_{i}$.

- The term $p_{j, i}(1, \ldots, 1) \cdot F_{g, i+1}$ is linear in $p_{i}$.

- The term $p_{j, i}(1, \ldots, 1) \cdot q_{g, i}$ is linear in $p_{i}$.

- The term $p_{j, i}(1, \ldots, 1) \cdot S_{g} r_{i}$ contains bilinear and linear terms in $p_{i}$.

These observations imply that equation (16) can be written as a scalar quadratic function in $p_{i}$.

$$
f_{j}\left(p_{i}\right)=0, j \in\{1, \ldots, \beta\}
$$

Assumption A8 implies that one of the partial pressures is linearly dependent on the others. Hence, the equations for computing the partial pressures in compartment $i$ can be formulated as a quadratic vector function

$$
\tilde{F}\left(p_{i}^{r}\right)=\left(\begin{array}{c}
f_{1}\left(p_{i}^{r}\right) \\
\vdots \\
f_{\beta-1}\left(p_{i}^{r}\right)
\end{array}\right)
$$

where $\tilde{F}: R^{(\beta-1)} \rightarrow R^{(\beta-1)}$, and $p_{i}^{r}$ denotes the vector with the partial pressures, except $p_{\beta, i}$, in compartment $i$.

The quadratic function $F$ will be analyzed closer later in this paper. 


\subsection{Assumptions and extensions}

In the above derivation we made four assumptions A5-A8. These will be discussed closer in the following. Assumptions A9-A12 will be elaborated on in the Integration Scheme section.

3.2.2. Stationary gas dynamics Normally the residence time for the gas within a vessel is far shorter than the residence time for the liquid and solids. To provide a concrete example the residence time for gas in an industrial $20 \mathrm{MW}$ furnace for ferroalloy or silicon production is less than $5 \mathrm{sec}$ while the residence time for the solids and liquids is several hours. Further, if the purpose of the model is to recreate the system dynamics, i.e. the dominant dynamics for the vessel, the gas dynamics can be approximated by A5. This is the reason for the widespread use of this assumption in numerical simulation.

3.2.2. Reaction kinetics We consider a single reaction where two components $A$ and $B$ react to $R$ and $S$, and assume constant volume.

$$
\eta A+\mu B \rightarrow \nu R+\xi S
$$

$\eta, \mu, v, \xi$ are positive integers given by the stoichiometry of the reaction The mole reaction rate $r$ will typically be determined by the concentration of the reactants $A$ and $B$ according to the following rate law

$$
r=k(T) n_{A}^{\varsigma} n_{B}^{\zeta}, \quad \varsigma \geqslant 0, \zeta \geqslant 0
$$

where $k(T)$ is defined by the Arrhenius equation $k(T)=A e^{-E / R T}$. If one of the components, say $B$, is in the gas phase, its partial pressure is proportional to the molar mass as shown in equation (12). In this case equation (20) can be rewritten.

$$
r=k^{\prime}(T) n_{A}^{\varsigma} p_{B}^{\zeta}
$$

Equations (20) and (21) should be viewed as ideal rate description laws. To exemplify deviations from this, a gas-solid reaction or a gas-liquid reaction in which the reaction takes place on the solid surface or liquid surface, the reaction rate is often controlled by the mass transport to and from the reacting surface. Another deviation arises when there is an excess of some component, e.g. gas in equation (21). In this case the concentration of gas does not influence the reaction rate. Hence, equation (21) may be reformulated to

$$
r=k^{\prime \prime}(T) n_{A}^{s}
$$

In many modeling problems there is a lot of uncertainty linked to the kinetics, especially in gas-solid systems. In such cases it very often suffices to choose the kinetics as linear in the partial pressure or independent of the partial pressure.

$$
r=k^{\prime \prime \prime}\left(n_{A}, T\right) p_{B}^{\zeta}, \zeta \in\{0,1\}
$$

We observe that equation (23) is a generalization of equation (11), i.e. the rate law used in the derivation of the general algorithm for computing the partial pressures. Hence, equation (11) may be generalized to

$$
r_{k, i}=f_{k}\left(n_{s, i}, T_{i}\right)\left(p_{l(k), i}-\bar{p}_{l(k)}\left(T_{i}\right)\right)^{\zeta k}, k \in\{1, \ldots, \gamma\}
$$

In the case where $\zeta_{k}=0$ the term $\Sigma_{k=1}^{\gamma} s_{g j, k} r_{k, i}$, cf. equation (15), is independent of $p_{i}$. Further, the term $p_{j, i}(1, \ldots, 1) \cdot \Sigma_{k-1}^{\gamma_{y j, k}} s_{k, i}$ will be linear in $p_{i}$. This implies 
that the corresponding function element $f_{j}$ in equation (17) will be a linear function in $p_{i}$.

In some cases the linear dependence on the partial pressure is too crude. This situation especially arises in bidirectional reaction schemes

$$
\eta A+\mu B \rightleftharpoons \nu R+\xi S
$$

with different reaction mechanisms in the two opposite directions. This may give a rate law that is piecewise linear in the partial pressure as shown below.

$$
r_{k, i}= \begin{cases}f_{k}\left(n_{s, i}, T_{i}\right)\left(p_{l(k), i}-\bar{p}_{l(k)}\left(T_{i}\right)\right) & \text { if } p_{l(k), i}>\bar{p}_{l(k)}\left(T_{i}\right) \\ g_{k}\left(n_{s, i}, T_{i}\right)\left(p_{l(k), i}-\bar{p}_{l(k)}\left(T_{i}\right)\right) & \text { if } p_{l(k), i} \leqslant \bar{p}_{l(k)}\left(T_{i}\right)\end{cases}
$$

This bidirectional kinetics law gives raise to more than one quadratic equation (18). If one rate law is piecewise linear, two independent quadratic equations will arise.

Equation (26) can be included in the derived method in a straightforward manner as shown later in equation (28).

In cases where the rate laws are nonlinear in the partial pressure(s) the derived methodology fails. Experience shows, however, that a linear approximation often suffices. Hence, the methodology may still be used in these cases.

3.2.3. The gas law A7 assumes an ideal gas. The additivity of the partial pressures and the partial volumes is based on the assumption that the gas molecules do not interact with eachother in the gas phase. This is a good assumption at low pressures, typically below 10 bar. It should be noted that $\mathrm{A} 7$ can be relaxed by introducing the compressibility factor in the equations of state, i.e. $p V=Z n R T$, where $Z$ may be a function of pressure, temperature and composistion. The use of the compressibility factor does not influence the derivation since $Z$ will be included in identically the same way as the right hand sides of equations (12) and (13).

3.2.4. Constant pressure The constant pressure assumption will be met if a vessel operates under atmospheric or close to atmospheric conditions, or if there is tight pressure control on the vessel. Tight pressure control means that the pressure control loop is much faster than the residence times of the solids and/or liquids. These are conditions that are satisfied in many industrially operated reactors or furnaces. Typical examples are electrochemical furnaces and blast furnaces, and pressurecontrolled reactors, e.g. polymerization reactors.

\subsection{Solving the quadratic equation}

It is well known that there are multiple solutions to a vector quadratic function. Some of these solutions will be non-physical. To avoid this equation (18) is changed so as to exclude non-physical solutions.

$$
\begin{aligned}
\tilde{F}\left(p_{i}^{r}\right) & =\left(\begin{array}{c}
f_{1}\left(p_{i}^{r}\right) \\
\vdots \\
f_{\beta-1}\left(p_{i}^{r}\right)
\end{array}\right)=0, p_{i}^{r} \in P_{\Sigma, i} \\
P_{\Sigma, i} & =\left\{p \in \mathscr{R}^{(\beta-1)}: p_{j} \in\left[0, p_{\Sigma, i}\right] \forall j \in\{1, \ldots, \beta-1\} \wedge \sum_{j=1}^{\beta-1} p_{j} \in\left[0, p_{\Sigma, i}\right]\right\}
\end{aligned}
$$


We are not able to rigorously show that this equation has one, and only one solution. It does, however, seem reasonable to believe that there is only one set of partial pressures, and hence one set of reaction rates, that satisfies equation (27) if the model description is based on sound physical knowledge of the process in question.

The coupled quadratic vector function equation (18) must be solved using an iterative algorithm. Newton algorithms use the inverse of the Jacobian. The Jacobian is defined by

$$
J(p):=\frac{d \tilde{F}(p)}{d p^{T}}
$$

An iterative Newton algorithm will have quadratic convergence close to the solution provided $J^{-1}(p)$ exists in an open set close to the solution, see e.g. Nocedal and Wright (1999). In practice a Newton algorithm may perform erratically far from the solution. Further the inverse $J^{-1}(p)$ may not exist or it may be ill-conditioned. Hence, some approximate Newton solution such as Broyden's method is usually applied. An approximate Newton method also has the advantage that the Jacobian need not be computed at every iteration, hence its computational load is lower than for the Newton method. This latter point is, however, not important in lowdimensional problems as will usually be the case for equation (27).

In the case with piecewise linear reaction rates equation (26) there will be a set of quadratic functions that are valid in subsets of $P_{\Sigma, i}$. Equation (27) should be formulated as follows:

$$
\begin{aligned}
\tilde{F}^{k}\left(p_{i}^{r}\right) & \left.=0, p_{i}^{r}\right\} \in P_{\Sigma, i}^{k} k \in\left\{1, \ldots, 2^{9}\right\} \\
P_{\Sigma, i}^{1} \cup \ldots \cup P_{\Sigma, i}^{2^{9}} & =P_{\Sigma, i}
\end{aligned}
$$

$\vartheta$ is the number of quadratic equations that are piecewise linear, cf. equation (26). Hence, if two rates are piecewise linear, four independent quadratic equations will arise.

Equation (28) is solved by solving the quadratic equations $\tilde{F}^{k}$ consecutively until a solution is found. In practice it is usually possible to solve the equations in an intelligent order in the sense that it may be known in which subset $P_{\Sigma, i}^{k}$ the solution most probably lies. This will obviously reduce the computational load.

A remark should be made to the case of two gas components $(\beta=2)$. In this case $p_{i}^{r} \in \mathscr{R}$, i.e. $\tilde{F}$ (or $\tilde{F}^{k}$ ) is a scalar quadratic function. Thus, there exists an explicit solution to $\tilde{F}\left(p_{i}^{r}\right)=0\left(\right.$ or $\tilde{F}^{k}\left(p_{i}^{r}\right)=0$ ), and hence, there is no need for an iterative solution algorithm.

\section{Integration scheme}

In this section we show how the derived theory can be used to construct a consistent and highly efficient integration method for a vessel divided into $N$ compartments. The exposition is based on one possible realistic configuration of a vessel. Alternative configurations will be discussed at the end of this section.

\subsection{Vertical vessel}

The vessel vertically is divided into compartments as shown in Figure 1. There is a (unidirectional) upwards flow of gas from compartment $N$ to 1 , and a (unidirectional) 
downwards solids and liquid flow from compartment 1 to $N$. We assume that there exists equations for the flow transport of solids and liquid. An example of such equations is based on the assumption that all vacant volume of a lower compartment immediately is filled by solids and liquid from the above compartment. The structure of these equations become:

$$
\begin{aligned}
& F_{s, i-1}=h_{i}\left(F_{s, i}, r_{i}, q_{s, i}\right), i \in\{2, \ldots, N-1\} \\
& F_{s, N-1}=h_{N}\left(q_{s, N}, r_{N}\right)
\end{aligned}
$$

Compartments 2 to $N$ all have constant volumes while the volume of compartment 1 may vary. The gas phase volume $V_{g, i}$ occupies a constant ratio of the volume of each compartment $V_{i}$.

Solids and/or liquid are fed into the vessel at compartment $1\left(q_{s, 1}\right)$ and gas escapes from the vessel at this point $\left(F_{g, 1}\right)$. Note that $F_{s, 0}=0, q_{g, 1}=0$. Solids and/or liquid are tapped from compartment $N$ meaning that $q_{s, N} \leqslant 0$. Note that $F_{s, N}=0$, $F_{g, N+1}=0, q_{g, N}=0$. The external power supply is set equal to zero $\left(Q_{i}=0\right)$, except the power supply to the lowest compartment $\left(Q_{N} \neq 0\right)$. The external flows, except $F_{g, 1}$, can either be specified as exogenous variables, or they may be controlled. As an example of the latter the power supply $Q_{N}$ may be controlled by the temperature in compartment $T_{N}$. Note that $F_{g, 1}$ is computed by the model.

The integration scheme at a time instant $t$ is given as follows:

1. The mass of solids and liquid components, the inner energy, the pressure, and the external solids and liquid flows, and power supply are given at time $t$, i.e. $n_{s, i}, U_{i}, p_{\Sigma, i} i \in\{1, \ldots, N\}, q_{s, 1}, q_{s, N}, Q_{N}$.

2. The temperatures $T_{i}, i \in\{1, \ldots, N\}$ are computed from thermodynamic equations or tables.

3. The equilibrium partial pressures are computed using $\bar{p}_{l(k)}\left(T_{i}\right) i \in\{1, \ldots, N\}$, $k \in\{1, \ldots, \gamma\}$ using the same thermodynamic tables as above.

4. Compute the partial pressures $\left(p_{N}\right)$ using equations (27) or (28), the reaction rates $\left(r_{N}\right)$ using equations (11) or (26), and the gasflow $\left(F_{g, N}\right)$ using equation (10) with $\dot{n}_{g, N}=0$ in compartment $N$.

5. Compute the partial pressures $\left(p_{i}\right)$ using equations (27) or (28), the reaction rates $\left(r_{i}\right)$ using equations equation (11) or (26), and the gasflow $\left(F_{g, i}\right)$ using equation (10) in compartment $N-1$ to 1 (in this sequence).

6. Compute the flowrate of solids and liquid $\left(F_{s, i}\right)$ for $i=\{N-1, \ldots, 2\}$ (in this sequence) using equations (30) and (29).

Items 1-6 provide the necessary input to compute the right hand side of the differential equations, equations (7) and (9).

Equations (7) and (9) are integrated, i.e. the states at the next time instant are computed, using an integration method for non-stiff systems, e.g. a Runge-Kutta method.

If the energy balances for each compartment is specified on a temperature explicit form, item 2 is not necessary since the compartment temperatures will be states. Hence, the tempearture will be specified in item 1 instead of the inner energy.

Linked to the initial section of this paper, in this vertical vessel example equations (7) and (9) are equivalent to equation equation (1), while equation (8) is equivalent 
to equation (6). $h$ in equation (3) is equivalent to equations (26), (27), (29) and (30) in addition to thermodynamic equations or tables. $z$ in equations (1), (6) and (3) include the temperatures $T_{i}$, the equilibrium partial pressures $\bar{p}_{l(k)}$, the partial pressures $p_{i}$, the reaction rates $r_{i}$, and internal solids and liquid flows $F_{s, i}$.

It is important to note that the merit of the proposed method is caused by the fact that equations (6) and (3) are solved in a sequential and decomposed way. In particular the computation of the partial pressures in item 4 and 5 above is critical for efficiency.

\subsection{Alternative vessels}

The method derived in section 3 can be used to numerically integrate alternative systems to the vessel system described above. The important issues are that the reaction rates, and the internal solids and liquid flow rates can be computed prior the integration the integration of equations (7) and (9).

Some alternative configurations are:

- Gas injection or gas withdrawal at one or several of the intermediate compartments.

- Solids and/or liquid injection or withdrawal at one or several of the intermediate compartments.

- External power supply to more than one compartment.

- Concurrent flow directions for solids and liquid, and gas.

- Time-varying compartment volumes.

\section{Case study}

The derived methodology will be highlighted by reporting on industrial experience using the methodology. Due to confidentiality issues only limited information can be supplied on the industrial case.

\subsection{Industrial experience}

The industrial case is based on a widely used reactor for metal production in the metallurgical industry, an electrochemical furnace for ferro-alloy production. Details can be found in Schei et al. (1998). The reactor is operated at atmospheric pressure. A dynamic model has been developed for the system. From a numerical integration point of view the model is very demanding. The ratio of the residence time for the gas compared to the solids is in the order of $10^{4}$. The demanding numerical integration problem has severely limited the use of earlier versions of the model Halvorsen (1993). The purpose of the model is to simulate time spans ranging from some hours to several weeks. The residence time for the solids is in the range of 110 hours.

A furnace has typically been divided vertically into 10 compartments $(N=10)$. Solids are fed into compartment 1 (cf. Figure 1), and gas escapes from this compartment to the environment. Liquid (metal and slag) is tapped from the bottom compartment $N$. Further, power $Q_{i}$ is supplied to all the compartments. The thermodynamic properties are computed using thermodynamic tables Chase (ed.) (1985).

The internal solids and liquid flows are based on the assumption that all vacant volume of a lower compartment immediately is filled by solids and liquid from the above compartment. 
The solution method has been applied to an industrially-applied simulator with excellent results. This is substantiated by the following: Results from extensive usage both by developers and users show that the integration scheme is extremely robust in the sense that it hardly ever fails, even if the model is simulated at operating conditions far from normal operating conditions, e.g. a start-up situation. This constitutes a major improvement as compared to earlier schemes, see e.g. Halvorsen (1993). Further, the integration scheme is fast. Typically 24 hours is simulated in 60 seconds on a $300 \mathrm{MHz}$ Pentium II. This also represents a major improvement (more than 10 times) compared to earlier methods. The numerical integration method has been a key factor for the success of the industrial simulator.

\subsection{Gas dynamics in the industrial model}

The furnace process has two gas components $(\beta=2)$ and four main reactions $(\gamma=4)$ which are linearly dependent on the partial pressures of the gas components. Since the total pressure is always close to one atmosphere in the real process, it is assumed that $\Sigma_{i=1}^{\beta} p_{i}=1 \mathrm{bar}$. Since only two gas components are considered relevant in the model, and the total pressure is known, the partial pressure of gas component 2 is given by $p_{2}=1-p_{1}$. Hence, the gas phase has only one unknown, $p_{1}$.

Each reaction rate varies proportionally to the distance from the reaction's equilibrium gas pressure, i.e. $p_{l(k), i}-\bar{p}_{l(k)}\left(T_{i}\right)$, or, alternatively, it is independent of the pressure. The rate equations are different, depending on the partial pressure being above or below the equilibrium pressure, see also equation (26). The reaction rates in the industrial model are all given as follows

$$
r_{k, i}= \begin{cases}k_{k, 1} n_{s j, i}\left(p_{l(k), i}-\bar{p}_{l(k)}\left(T_{i}\right)\right) & \text { if } p_{l(k), i}>\bar{p}_{l(k)}\left(T_{i}\right) \\ k_{k, 2} n_{s, i}\left(p_{l(k), i}-\bar{p}_{l(k)}\left(T_{i}\right)\right) & \text { if } p_{l(k), i} \leqslant \bar{p}_{l(k)}\left(T_{i}\right)\end{cases}
$$

where $k_{k, 1}, k_{k, 2}$ are the rate constants for the forward and backward reaction, and $n_{s j, i}$ is the amount of one of the solid or liquid components in compartment $i$, cf. equation (21).

There are four equilibrium lines within the two-dimensional $p_{1}-T$ space. Hence, this space may be divided into $2^{4}=16$ disjunct regions. Within each region we construct one quadratic equation in the unknown partial pressure, cf. equations (27) and (28). Since there is one unknown partial pressure $\left(p_{1}\right)$, the 16 quadratic equations are scalar equations.

Since the reaction rates are different above and below the equilibrium pressure, all 16 quadratic equations must in principle be checked for a solution. Since some of the regions are non-physical, the actual number of equations is lower. Further, note that the definition of $P_{\Sigma, i}$ equation (27) ensures that the algorithm chooses the physically meaningful solution of any of the 16 quadratic equations.

\subsection{Case simulation}

In this section we will highlight the performance of the numerical method by showing some simulation results.

The most critical scenarios in terms of model robustness and numerical robustness is when the model state changes rapidly over a wide operating range, often out of the normal operating area. An example of such a scenario is furnace start-up or shut down. Then the partial pressures in the gas phase typically change rapidly, as does 
the temperatures when the power is turned on and off. Below we show how the model, using the described calculation scheme for the gas dynamics, during a series of rather extreme changes in operating conditions. The results are illustrated in Figures 2 and 3.

Initially, the furnace is operating under normal conditions. After 4 hours of simulation, the furnace power is turned off. The gas pressure drops radically. At 10 hours, the furnace power is turned on again. The changes are again quite profound.

At 19 hours, $50 \%$ of all the gas produced in compartment $N$ is allowed to escape directly out of the compartment ( $q_{g, N}$ changes in a stepwise manner from 0 to a large negative value) without flowing through the compartments above. This is a radical change, which is not likely to happen to such a large extent in the real process. The gas compositions in the above compartments are heavily influenced by the change, while compartment $N$ is less affected. At 28 hours of simulation the leakage is stopped, and the process slowly works its way back to normal working conditions. The slow return of the partial pressures is caused by severely deteriorated furnace conditions due to the $50 \%$ leakage of gas from compartment $N$. This is in agreement with what is expected to happen on a real process.

At 76 hours, a vital reaction is turned off. This has dramatic consequences for the process. At 85 hours the reactions is turned back on. The process is now, as should be expected, far off the normal operating conditions, and will be difficult, if not impossible to bring back to normal operation.

Through all the rather extreme changes of the above scenario, the method computes the fast gas dynamics with a remarkable robustness. This simulation has

\section{p_1,Comp5 [solid line], p_1,CompN [dashed line]}

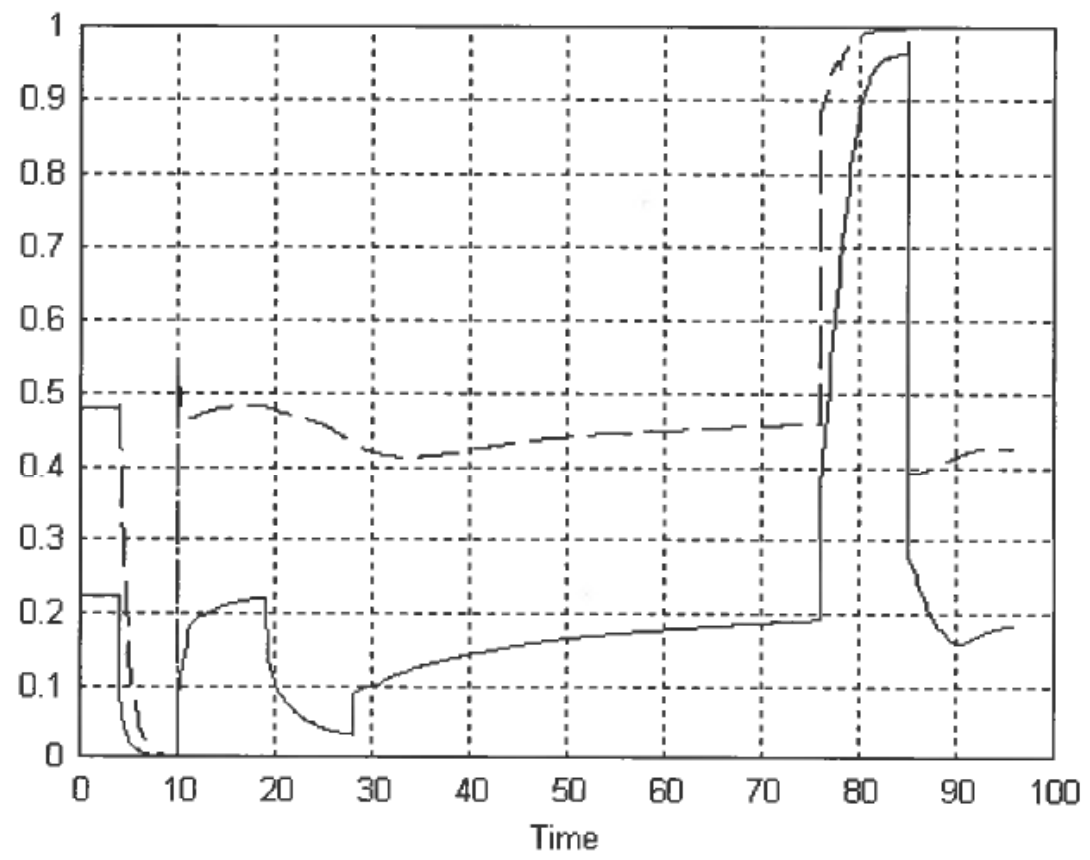

Figure 2. Partial pressure of gas component 1 in compartment 5 and compartment $N$ $\left(p_{1,5}, p_{1, N}\right)$. 


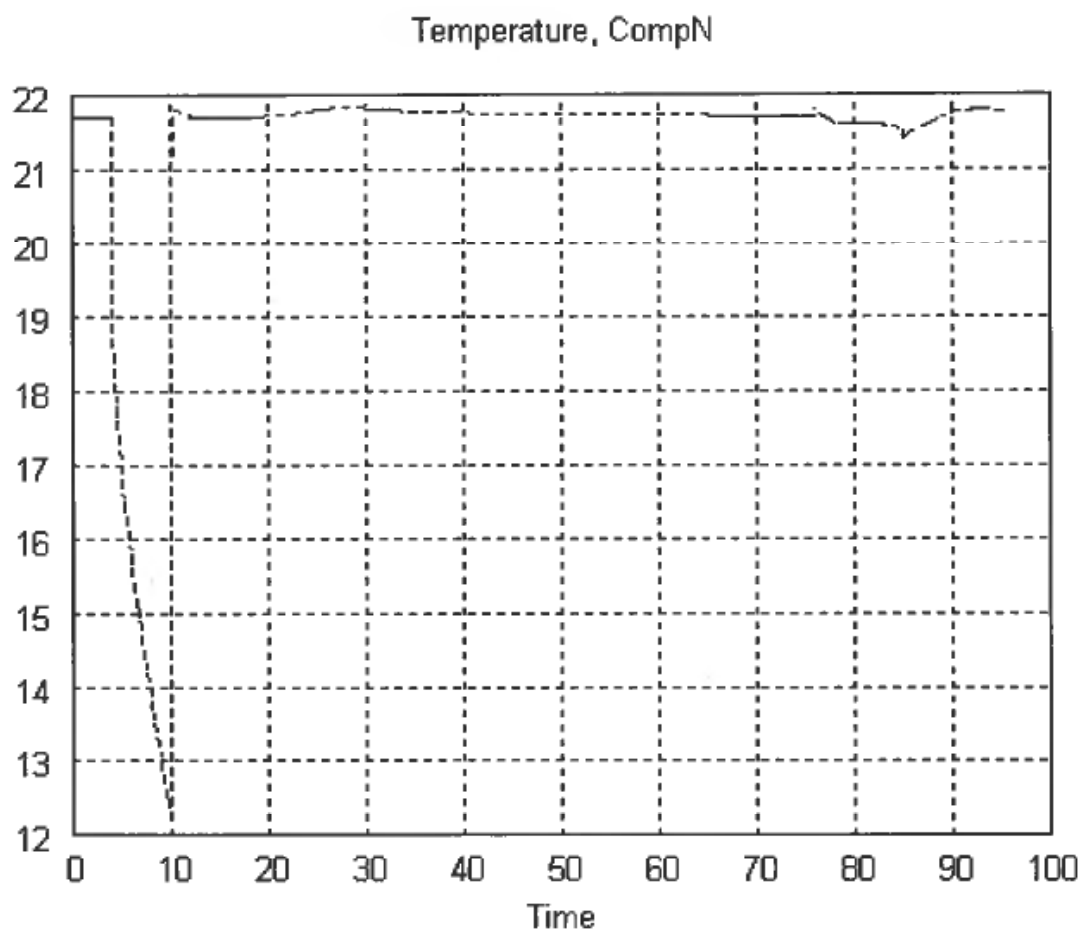

Figure 3. Temperature in compartment $N\left(T_{N}\right)$.

been run using an ordinary explicit Runge-Kutta 4-5 method to integrate equations (7) and (9). Compared to the advanced numerical integration methods used in previous model implementations, this is quite remarkable.

The robustness and speed of the simulation method is greatly appreciated as the model can be simulated several times faster than real-time, and without the danger of 'crashing' the simulation when the model state is out of the normal operation range.

\section{Conclusions}

In this work we have developed an efficient and robust integration method which is suitable for a large class of industrially relevant systems. The methodology has in practice performed excellent and been a key factor for the success of the industrial simulator for electrochemical furnaces for ferro-alloy production.

\section{Nomenclature}

$E$ - activation energy

$\tilde{F}$-quadratic equation in the partial pressure

$F_{g, i}^{T}=\left[F_{g 1, i}, \ldots, F_{g \beta, i}\right]$--gas flow from compartment $i$

$H_{g}^{T}=\left[H_{g 1}, \ldots, H_{g \beta}\right]-$ enthalpy content in gas phase

$H_{s}^{T}=\left[H_{s 1}, \ldots, H_{\mathrm{sa}}\right]-$ enthalpy content in solids and liquid phase

$\Delta H^{T}=\left[\Delta H_{1}, \ldots, \Delta H_{\gamma}\right]$-reaction enthalpy $i$ to compartment $i-1$

$J$-Jacobian matrix 
$n$-mass in moles

$n_{g, i}^{T}=\left[n_{g 1, i}, \ldots, n_{g \beta, i}\right]$-vector with gas components in moles in compartment $i$

$n_{s, i}^{T}=\left[n_{s 1, i}, \ldots, n_{s x, i}\right]$-vector with solids and liquid components in moles in compartment

$p$-pressure

$p_{i}^{T}=\left[p_{1, i}, \ldots, p_{\beta, i}\right]$-vector with the partial pressures in compartment $i$

$p_{i}^{r T}=\left[p_{1, i}, \ldots, p_{\beta-1, i}\right]-$ vector with the partial pressures except $p_{\beta, i}$ in compartment $i$

$p_{\Sigma, i}$-pressure in compartment $i$

$\bar{p}_{l(k)}$ - equilibrium pressure of gas component $l$ for reaction $k$

$Q_{i}$ - power supply to compartment $i$

$R$ - the universal gas constant

$r_{i}^{T}=\left[r_{1 . i}, \ldots, r_{\gamma, i}\right]$-vector with the reaction rates in compartment $i$

$S_{g}=\left\{s_{g l, m}\right\} \in R^{\beta} \times R^{\gamma}$-stoichiometry matrix for the gas components

$S_{s}=\left\{s_{s l, m}\right\} \in R^{\alpha} \times R^{\gamma}$-stoichiometry matrix for the solids and liquid components

$t$ - time

$T$-temperature

$U_{i}$-inner energy in compartment $i$

$V_{i}$-volume of compartment $i$

$V_{g, i}-$ volume of gas phase in compartment $i$

$V_{s, i}$-volume of solids and liquid in compartment $i$

$x$ - state variables

$y$ - state linked to fast dynamic modes

$Z$ - compressibility factor in the equation of state

$z$ - auxiliary variables

$\alpha$-total number of solids and liquid components

$\beta$ - total number of gas components

$\gamma$-total number of reactions

$\vartheta$-number of reactions that are piecewise linear

\section{Acknowledgement}

We thank Elkem ASA (www.elkem.com) for allowing us to use one of their models as a case study, and Ms. Lisbeth Hvidsten for contributions during her diploma work.

\section{REFERENCES}

Aitken (ed.), R. C. (1985). Stiff Computation. Oxford University Press

Chase (ed.), M. W. (1998). Nist-janaf thermodynamic tables-4th ed. J. Phys. Chem. Ref. Data. de Swart, J. J. B., Hoffman, J. E., Lioen, W. M. and Sommeijer, B. P. (1999). Test set for ivp solvers. Technical report. CWI publication.

Fogler, H. S. (1986). Elements of Chemical Reaction Engineering. Prentice-Hall.

HaIrer, E. and WanNer, G. (1999). Solving Ordinary Differential Equations II-Stiff and Differential-Algebraic Systems. Springer Verlag.

Halvorsen, S. A. (1993). Mathematical model(s) for the (ferro)silicon process. In: Proc. ECMI 93. Montecatini Terme, Italy. 
LAMBERT, J. D. (1991). Numerical Methods for Ordinary Differential Systems. Wiley.

LEVENSPIEL, O. (1972). Chemical Reaction Engineering. Wiley.

Michelsen, F. A. and Foss, B. A. (1996). A comprehensive mechanistic model of the continuous kamyr digester. Applied Mathematical Modelling 20, 523-533.

Nocedal, J. and Wright, S. J. (1999). Numerical Optimization. Springer-Verlag.

SCHEI, A., Tuset, J. K. and Tvert, H. (1998). Production of High Silicon Alloys. Tapir, Norway. 